\title{
Integration of internet-based genetic databases into the medical school pre-clinical and clinical curriculum
}

Darrel J. Waggoner, $M D^{1}$, and Christa Lese Martin, $P h D^{1,2}$

Over the past several years, the field of medical genetics has continued to expand and is now impacting a broad range of medical care, mainly due to rapid advances in genetic technology and information generated by the Human Genome Project. Physicians from multiple disciplines will need to become familiar with genetic principles, and the availability of genetic databases on the internet is a valuable resource for medical students and physicians.

To integrate these tools into medical student training, the University of Chicago Pritzker School of Medicine set out to develop multiple, interactive, case-based, educational sessions in the pre-clinical and clinical curriculum, designed to reinforce basic principles taught in the pre-clinical genetics class and demonstrate the usefulness of genetic information accessible via the internet in the clinical setting.

Two interactive sessions and a self-assessment exercise were developed. The sessions took place in a computer classroom where each student had access to the internet and could work independently. The sessions used case-based scenarios to help students become familiar with internet based resources and demonstrate how genetic information can affect medical care.

The sessions were well received by the student participants with $99 \%$ agreeing that the material was useful and important to clinical medicine. In a follow-up questionnaire $1 / 3$ of the students reported using the databases presented during class in a clinical setting. Genet Med 2006:8(6):379-382.

Key Words: internet resources, interactive learning, genetics

Information generated by the Human Genome Project has revolutionized medical research and is in part responsible for the blurring of traditional boundaries of basic science disciplines. Similarly, the practice of medicine is being affected by new genetic discoveries as well. Medical genetics and diagnostic genetic testing have progressed from the study of rare diseases to impacting all areas of clinical medicine, from primary care to subspecialty practice. The influence of medical genetics will only continue to grow as the understanding of the genetic basis of complex traits, such as obesity and heart disease, improves. In addition, advances in biotechnology, such as the development of microarrays, have already resulted in the ability to do large-scale sequencing, characterize genome-wide sequence variations and genomic imbalances and describe tumor grading by gene expression profiling. ${ }^{1,2}$ As the etiology of more diseases are defined on a molecular basis and genetic testing techniques become more sophisticated, affordable and

From the ${ }^{1}$ Department of Human Genetics, University of Chicago, Chicago, IL; ${ }^{2}$ Department of Human Genetics, Emory University, Atlanta, GA.

Darrel J. Waggoner, MD, University of Chicago, Department of Human Genetics, 5841 South Maryland Avenue Room L155, MC0077 Chicago, IL 60637.

Submitted for publication December 28, 2005.

Accepted for publication March 29, 2006.

DOI: 10.1097/01.gim.0000223543.63104.5a practical, the use of diagnostic genetic testing will become routine for many primary and subspecialty physicians.

This explosion of medical genetic information and technology has been accompanied by the development and routine use of databases, especially those available via the internet. As shown in Table 1, these databases and internet sites contain valuable information related to specific diseases, DNA sequences, and genetic testing which are important resources available to physicians, patients, and families.

In tandem with the rapid growth and heightened impact of the medical genetics field comes the recognition that many established physicians have had little, if any, formal education or training in medical genetics. Thus, many doctors are insufficiently trained to order and interpret genetic tests as well as to counsel patients about the results. Although the best mechanism to deliver genetics education to physicians who have completed medical training remains unclear, genetics curriculum is being developed and integrated into most medical schools. The traditional model for most of these courses is a combination of lectures and small group discussions in the first or second year of basic science course work. However, there is a paucity of educational curricula or tools introduced during the clinical years. ${ }^{3}$ In addition, there is little information about the incorporation of internet resources into the formal curriculum. 
Table 1

Internet databases

National Center for Biotechnology Information

www.ncbi.nlm.nih.gov/

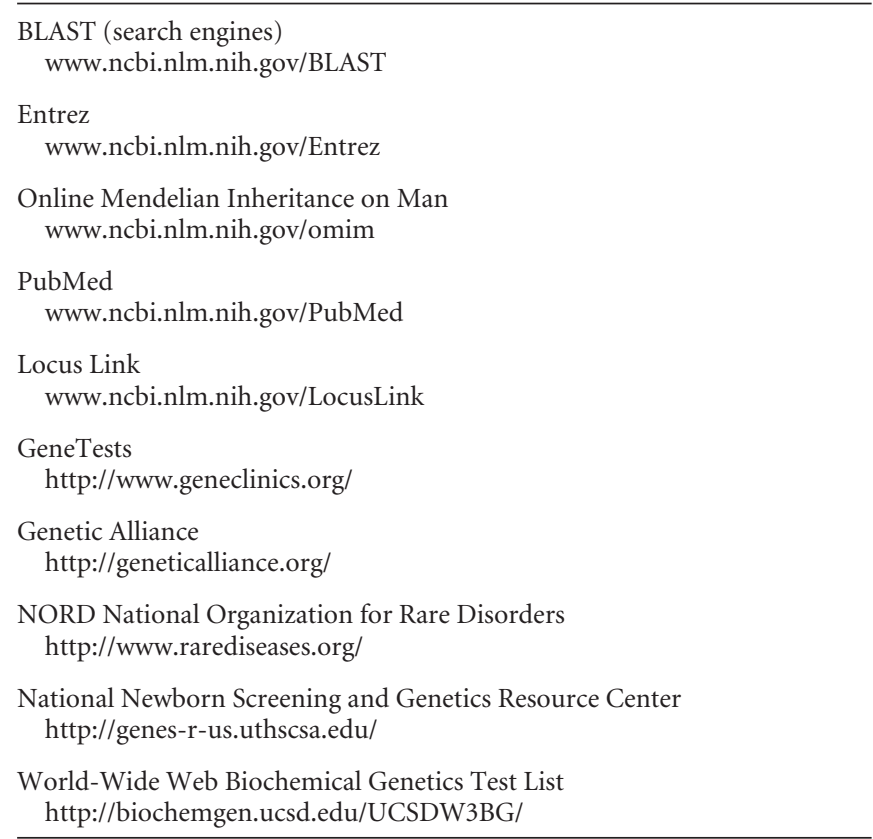

In recognition of the growing role of molecular genetics, cytogenetics and medical genetics in clinical medicine, the Information and Education Committee of the American Society of Human Genetics (ASHG) has developed recommendations for medical school curricula, which include the use of computer based resources. ${ }^{4}$ Despite these recommendations, there are few published examples of medical school curricula that address these objectives.

\section{DEVELOPMENT AND INTEGRATION OF GENETIC BIOINFORMATIC SESSIONS}

The Pritzker School of Medicine at the University of Chicago has recently developed two bioinformatics educational sessions and incorporated them into the first-year medical genetics course (Session One) and the third-year pediatrics clinical rotation (Session Two). In each of these sessions, students meet in a computer training classroom where each student has access to a computer with an internet connection and the session leader has a computer with projection capabilities. The students are given practical problems related to scientific questions in the first year and clinical questions in the third year. The group leader discusses the various internet-based genetic resources while demonstrating how answers to the questions can be obtained. The students then use the databases at their own computer to complete the problem set. These sessions reinforce the basic genetic principles taught in the first-year medical genetics course and demonstrate the use of these resources in patient care during the pediatric rotation.

Session One was designed similarly to the model developed at Washington University School of Medicine ${ }^{5}$ where a module was added within the medical genetics course to teach students how to access information from various internet resources by using clinical case vignettes. Session One is designed to introduce students to the databases available at the National Center for Biotechnology Information (NCBI) Web site (Table 1) to help them gain a better appreciation of the data generated by the human genome project and its applications. Prior to the session, the students are provided a handout that reviews the NCBI site, the databases that are available through this site and a short tutorial on how to navigate and utilize these resources.

For this module students were given a sequence fragment containing a mutation which corresponded to the Menkes disease gene, ATP7A (Table 2). The report by Washington University ${ }^{5}$ contains helpful information for developing a sequence and in our experience careful attention to this detail prior to the session was crucial. Using the NCBI programs

\section{Table 2}

Questions for session one (including DNA sequence for ATP7A with mutation $2936 \mathrm{c}>\mathrm{t}$ ) and session two

Session One

attgtggaaacctactttcctggctacaatagaagtatctcctgaacagaaacgataatacgatttgctttccaagcctc

Identify the gene for this sequence.

Identify the disease caused by mutations in this gene.

Identify and name the specific mutation at the DNA level.

Identify and name the specific mutation at the amino acid level.

Why is this mutation pathogenic?

Identify any additional disease(s) associated with mutations in this gene.

What is the normal function of this protein?

Are there any treatment options available for a patient with this mutation?

Are there any homologous human genes, and if so do mutations cause a different disease, and if so why?

Session Two

CASE 1: You admit a 6-month-old patient who presents with fever and cloudy urine and is admitted for treatment of a urinary tract infection (UTI) and possible sepsis. History reveals that he has a known kidney malformation (the mom can't remember the details) and has had UTI in the past. In addition, you note on exam that he has a hypoplastic underdeveloped thumb. The mom is 10 weeks pregnant and is seeking a diagnosis for her child and inquires about the chance that any similar problems could occur in her next child. After initiating therapy for the infection and making sure the patient is stable, how do you proceed at obtaining a diagnosis? After this review you go back and reexamine the child and note that he is small, has a small head, and has several café au-lait-spots. How would you proceed with diagnostic testing based on your clinical suspicions? What could you tell mom about recurrence risks?

CASE 2: You admit a child to the floor post-operatively who has had a mandibular distraction by the pediatric plastic surgery service. The child carries a diagnosis of Treacher Collins syndrome. Are there any specific issues you should be worried about or address with the family?

CASE 3: You are seeing a 2-month old infant in your practice who just moved to Texas from Nevada. Where on the Internet can you go to determine what newborn screening tests are routinely done in Nevada?

CASE 4: A 3-month-old child with a diagnosis of achondroplasia is admitted for a history of apneic episode at home. What should you do and is this related to the achondroplasia?

CASE 5: You are taking care of a child in the NICU (neonatal intensive care unit) with onset of seizures at birth. 
blastn and blastx, the students are asked to identify the gene corresponding to this sequence, the disease caused by mutations in this gene, and the mutation at the DNA and amino acid level. This exercise allows an introduction to bioinformatics and its application in clinical genetics and genetics research. Identifying the mutations at the DNA level (the numbered base pair and change) and the amino acid level (the numbered amino acid and change) reinforces basic concepts taught in class such as utilizing mutation nomenclature, determining mutation types (missense, nonsense, insertion, deletion, etc.), and differentiating between coding sequence versus genomic sequence (used in identifying the position of the base pair mutated).

In addition, the students are asked to identify the diseases caused by mutations in this gene, determine the normal function of the gene, and speculate on the molecular pathophysiology of the disease and treatment options based on this understanding. This set of questions introduces the databases Entrez, OMIM, and PubMed. Reviewing the normal function of the gene and the molecular genetics of the disease reinforces the concepts of the molecular pathophysiology and possible treatment alternatives. The PubMed review strengthens the discussion by demonstrating clinical and treatment information.

Finally, students are asked to identify homologous genes and diseases associated with these genes. In this example, the $A T P 7 B$ gene (Wilson disease) should be identified through blastn and blastx. These questions promote discussion about the usefulness of homology searches between species, gene families, and the various uses of Entrez. The molecular basis of disease is again reviewed in this example with respect to mutations in homologous genes with identical function leading to distinct phenotypes based on tissue expression differences.

This session is followed up with a self-assessment, take home problem set, administered via the internet. The University of Chicago utilizes an electronic resource which allows the students to access, complete, and submit the problem set via the internet. The take-home problem set includes questions similar to the ones covered in Session One. Additional questions include ones that require students to design a molecular-based test to detect the mutation present and to discuss the advantages and limitations of the various molecular techniques chosen. They are also asked how they would counsel the patient and family regarding recurrence risks, which requires them to obtain additional information from the electronic databases to recognize issues related to germ-line mosaicism and, in this particular example, an advanced paternal age affect. The selfassessment is also designed to reinforce the concepts presented in class as well as to demonstrate how assimilation of the information available from the internet databases can be useful in interpreting genetic test results and communicating with families. Specific medical questions are also presented to help the students develop the skills of self-directed learning and problem solving related to clinical medicine.

Session Two was designed to enhance vertical integration of genetics in the medical school curriculum. Third-year students are introduced to databases available via the internet which can be used to assist in patient evaluations, diagnosis, and genetic counseling. Multiple clinical scenarios are presented with associated problem sets to facilitate discussion (Table 2). The first scenario presents several clinical and physical examination findings from a patient and the students are asked to identify a possible syndromic diagnosis which could explain this presentation. OMIM is used to identify diagnostic possibilities and the features of OMIM and its uses are reviewed. Inheritance patterns and counseling regarding recurrence risks are built into the question to review these concepts introduced in the first year genetics class. GeneClinics is used to find access to genetic testing and facilitate a discussion regarding the medical, family, insurance, consent, and ethical issues surrounding such testing. In addition, the differences between research and clinical testing are reviewed.

A second scenario regards the treatment of a patient with a rare syndrome and is specifically aimed at helping the parents to understand their child's disease. The National Organization for Rare Diseases (NORD) and Genetic Alliance websites (Table 1) are highlighted as places for access to disease specific information for rare diseases and the concept of family support groups and social support is introduced. Other scenarios are used to review biochemical diseases and newborn screening that incorporate the use of websites that give information on the diseases screened in each state. Finally, a clinical question regarding achondroplasia and apnea in the newborn period facilitates discussion not only related to the specific details of this disease, but also broaches the broader topic of using access to these databases to facilitate and improve medical care.

\section{STUDENT EVALUATIONS OF THE SESSIONS}

Both sessions were well-received by the first- and third-year students and highly evaluated. Session One was evaluated as part of the overall medical genetics class evaluation, which is done online at the Pritzker School of Medicine. A specific evaluation form for Session One was used and over a two-year period, 100/210 (48\%) students completed the evaluation. Fiftyeight percent of the students indicated they were not previously familiar with NCBI or any of the other databases. Additional responses were reported using a scale of strongly agree, agree, neutral, disagree or strongly disagree. With regard to the perceived usefulness of the session from a medical and scientific perspective, $58 \%$ of the students strongly agreed and $41 \%$ agreed and $98 / 100$ (98\%) students indicated the session should be kept in the class and repeated the following year. With regard to the importance of using a computer training classroom to facilitate the session, $75 \%$ of the students strongly agreed and $20 \%$ agreed that the computer classroom was helpful. Overall, the student comments were very positive, even from students with a scientific background who were already familiar with the NCBI site.

Session Two was evaluated immediately following the session. Eighty-two of 113 (73\%) students strongly agreed and 29/113 (26\%) agreed that Session Two was useful from a medical and clinical perspective. When asked, "Will you incorporate any of this information into your clinical practice?", 61/ $114(54 \%)$ strongly agreed and 48/114 (42\%) agreed. With 
regard to whether Session Two presented new information compared to Session One, 69/112 (62\%) strongly agreed and $39 / 112$ (35\%) agreed. Finally, with regards to the usefulness of the computer training classroom, 77/114 (67\%) strongly agreed and 23/114 (20\%) agreed that this type of classroom is helpful.

Students who completed Session Two and were either in the remaining third-year rotations or had started their fourth-year training were contacted for follow-up evaluation to assess how often they used the bioinformatics tools. Twenty-five students responded to the evaluation. Eighteen of 25 (72\%) indicated they used OMIM anywhere from 1-10 times in different clinical settings including pediatrics, family medicine, surgery, and internal medicine rotations. Eight of $25(32 \%)$ had used GeneClinics to investigate genetic testing possibilities. This number is in contrast to 28 students who were contacted who had not yet participated in Session 2: none of them had ever used OMIM or GeneClinics in their clinical practice. Interestingly, only $6 / 53(11 \%)$ students contacted for follow-up indicated they had ever seen a resident or attending physician use any of these internet sites in practice.

\section{DISCUSSION}

The advances of genetic technology, genomics, and bioinformatics are changing the face of medicine and expanding the utility of medical genetics. Educational innovations are needed that keep pace with these advances and give students the building blocks that will allow them to keep abreast of these devel- opments. Public databases allow ready access to this information, but students and physicians need to be alerted to these resources, understand how they are organized, and be willing to access the information in order to incorporate the data into their practice.

The modules described here were successful in introducing students to genetic databases in the basic sciences course and in the clinical arena. Based on student feedback, both sessions were well received and the students performed well on the take home problems sets. The follow-up feedback was encouraging in that many students reported use of these databases after the clinical session, while no student who has not taken the session has ever used any of the databases. It is discouraging that the students do not see the residents or attending physicians using these resources and therefore the concepts learned in class are not being reinforced. As a start, the modules introduced here are relevant and useful additions to medical school curricula.

\section{References}

1. Obeid PJ, Christopoulos TK. Microfabricated systems for nucleic acid analysis. Crit Rev Clin Lab Sci 2004;41:429-465.

2. Sorlie T. Molecular portraits of breast cancer: tumor subtypes as distinct disease entities. Eur J cancer 2004;40:2667-2675.

3. Korf BR. Integration of genetics into clinical teaching in medical school education Genet Med 2002;4:S33-S38.

4. ASHG Report: Report from the ASHG Information and Education Committee: Med ical School Core Curriculum in Genetics. Am J Hum Genet 1995;56:535-537.

5. Magee J, Gordon JI, Whelan A. Bringing the human genome and the revolution in bioinformatics to the medical school classroom: A case report from Washington University School of Medicine. Acad Med 2001;76:852-855. 